\title{
Explicit Memory Performance in Infants of Diabetic Mothers at 1 Year of Age
}

\author{
Tracy DeBoer \\ Institute of Child Development and Center for Neurobehavioral Development, University of \\ Minnesota
}

Sandi Wewerka

Institute of Child Development, University of Minnesota

Patricia J. Bauer

Institute of Child Development and Center for Neurobehavioral Development, University of Minnesota

Michael K. Georgieff and Charles A. Nelson

Department of Pediatrics, Institute of Child Development, and Center for Neurobehavioral

Development, University of Minnesota

\section{Abstract}

The aim of the present research was to investigate the impact of abnormal fetal environment on explicit memory performance. Based on animal models, it was hypothesized that infants of diabetic mothers (IDMs) experience perturbations in memory performance due to exposure to multiple neurologic risk factors including: chronic hypoxia, hyperglycemia/reactive hypoglycemia, and iron deficiency. Memory performance, as measured by the elicited/deferred imitation paradigm, was compared between 13 IDMs ( 7 female, 6 male; mean age 365 days, $S D$ 11) and 16 typically developing children ( 7 female, 9 male; mean age 379 days, $S D$ 9). The IDM group was characterized by shorter gestational age (mean 38 weeks, $S D 2$ ), greater standardized birth weight scores (mean 3797 grams, $S D$ 947), and lower iron stores (mean ferritin concentration $87 \mu \mathrm{g} / \mathrm{L}, S D$ 68) in comparison with the control group (mean gestational age: 40 weeks, $S D$ 1; mean birth weight: 3639 grams, $S D$ 348; mean newborn ferritin concentration $140 \mu \mathrm{g} / \mathrm{L}, S D$ 46). After statistically controlling for both gestational age and global cognitive abilities, IDMs demonstrated a deficit in the ability to recall multi-step event sequences when a delay was imposed. These findings underscore the importance of the prenatal environment on subsequent mnemonic behavior and suggest a connection between metabolic abnormalities during the prenatal period, development of memory circuitry, and behavioral mnemonic performance.

\begin{abstract}
Infants of diabetic mothers (IDMs) comprise a compelling group in which to examine associations between fetal risk factors and subsequent memory performance. The adverse environment associated with the diabetic pregnancy consists of multiple neurologic risk factors including: 1) chronic hypoxia (Widness et al., 1981), 2) hyperglycemia/reactive hypoglycemia, and 3) iron deficiency (Petry et al., 1992), which, on the basis of animal models, have been shown to act selectively on regions of the fetal brain that are involved in explicit memory (e.g., the hippocampus, Barks et al., 1995; de Ungria, et al., 2000). Based on the known
\end{abstract}

Address comments and questions to Charles A. Nelson, Institute of Child Development, 51 East River Road, Minneapolis, MN 55455-0345, USA. Fax: (612)625-1530. canelson@umn.edu.. 
pathophysiology, it is hypothesized that exposure to these risk factors during the prenatal period may alter memory performance in human infants (see Georgieff \& Rao, 2001 for review).

Several studies have demonstrated that neurobehavioral outcomes in human children who were born to diabetic mothers are inversely correlated with the quality of metabolic regulation during pregnancy (e.g., Rizzo et al., 1997). However, these investigations have typically examined global cognitive development in children well downstream from the proposed insult, and are not necessarily related to specific risk factors or areas of injury. The purpose of the present research was to further characterize outcomes related to development in an abnormal prenatal environment by examining the relation between IDM's abnormal prenatal environment and behavioral explicit memory performance using the elicited imitation paradigm.

\section{Risk Factors}

Prenatal hypoxia and hyperglycemia/reactive hypoglycemia have been associated with both poor behavioral and neurologic outcomes. Specifically, prenatal hypoxia is linked with motor and cognitive deficits in humans (e.g., Low et al., 1984) and damage to memory areas such as the cerebral cortex, striatum, and hippocampus in animal models (e.g., Nelson \& Silverstein, 1994). Severe postnatal hyperglycemia and reactive hypoglycemia are linked with impairments in both cognitive functioning and learning in humans (e.g., Hannonen et al., 2003), and animal models have demonstrated that certain regions of the brain (i.e., the hippocampus) show particular vulnerability (Barks et al., 1995). When these risk factors co-occur, as in the diabetic pregnancy, oxygen consumption of the fetus increases and renders the fetus hypoxemic (Widness et al., 1981), which can ultimately result in brain iron deficiency through shifting of available fetal iron away from the brain and into the expanding red cell mass (Petry et al., 1992). In short, gestational diabetes mellitus introduces multiple risk factors to the developing fetus, each of which has been shown to have independent effects on neurodevelopment and thus may impact mnemonic behavior.

\section{Elicited Imitation}

Elicited/deferred imitation involves using novel toys to produce an action or a sequence of actions that the infant imitates. It is generally accepted as a nonverbal analogue to hippocampally-mediated verbal recall and has been used in numerous studies to assess explicit memory abilities in preverbal infants (Bauer, in press). One of the most compelling arguments in support of the analogy is the finding that amnesic adults who sustained damage to the hippocampal region are unable to perform an age-appropriate version of the task (McDonough et al., 1995); developmental amnesiacs show similar deficits (Adlam et al., in press; see Bauer, in press, for further developments of the analogy).

The general elicited imitation paradigm can be modified to produce a battery of nonverbal mnemonic tasks resembling several explicit memory tasks in the adult: 1) immediate imitation as an index of short-term recall, 2) deferred imitation as an index of long term recall mediated by the hippocampally dependent explicit memory system, and 3) interleaved presentation as a measure of working memory (see Bauer, in press for further elaboration). Immediate imitation performance is subject to rapid decay and does not correlate with recall performance following delays (10 minutes to 48 hours in length; Bauer et al., 1999); therefore, immediate imitation is argued to be mediated by a short-term memory store, represented by a temporary pattern of activation. In contrast, delayed imitation is mediated by a representational system that requires the transfer of the information from short-term storage into something more durable, such as the hippocampally-mediated memory system. Finally, when the steps of one sequence are presented interspersed with steps from another sequence (i.e., the presentation of the to-beremembered sequences is interleaved) a working memory requirement is imposed and the 
infant must hold in mind the individual steps of the events as they integrate the information over time.

\section{Present Investigation}

In the present investigation we examined whether 1) IDMs exhibited deficits in explicit memory performance at the end of the first year of life, 2) any observed deficits were specific to explicit memory or were pervasive across general memory processes, and 3) if any of the specific risk factors appeared to be associated with memory ability.

\section{Method}

\section{Participants}

Forty-three 12-month-old infants (29 control, 14 IDM) were enrolled in a longitudinal research project investigating prenatal risk factors on cognitive development participated. The sample consisted of predominately Caucasian infants $(91 \%)$ born to families of middle to high socioeconomic status (see DeBoer et al., 2004; deRegnier et al., 2000; Georgieff et al., 2002; Nelson et al., 2000; Nelson et al., 2003; Siddappa et al., 2004 for other reports on this sample).

Pregnant women were recruited at 28 weeks gestation from United Hospital Children's Hospital of St. Paul, University of Minnesota/Fairview Riverside Medical Center, and AbbottNorthwestern Hospital. In addition to meeting the medical criteria outlined in Table 1, infants born to women diagnosed with diabetes mellitus were eligible for inclusion in the IDM sample if they were delivered at 32 weeks gestation or more and were the products of an otherwise uncomplicated neonatal course. Infants in the control group were eligible if they were products of pregnancies uncomplicated by diabetes, were of 36-41 weeks gestation, had a birth weight that was appropriate for gestational age. At time of delivery, infants were assessed for signs of iron deficiency via cord serum ferritin concentrations and exposure to hypoxia and hyperinsulinemia via neonatal macrosomia (Akin et al., 2002;Morris et al., 1985). Ferritin values less than $76 \mu \mathrm{g} / \mathrm{L}$ were considered deficient in fetal iron stores (Tamura et al., 2002) and size for dates (i.e., birth weight $\mathrm{z}$ scores) greater than 2 standard deviations above the population mean suggested the presence of chronic fetal hypoxia and hyperinsulinemia (Nold $\&$ Georgieff, 2004). Eleven control infants were excluded from the sample due to unknown $(n=5)$ or low $(<76 \mu \mathrm{g} / \mathrm{L} ; n=6)$ ferritin concentrations. Comparisons between the groups indicated that the IDM group had greater birth weight $\mathrm{z}$ scores $(M=1.8, \mathrm{SD}=2.1)$ than the control group $(M=.43, \mathrm{SD}=.75), F(1,27)=6.05, p<.05$, and lower newborn ${ }^{1}$ ferritin concentrations $(M=87 \mu \mathrm{g} / \mathrm{L}, S D=68)$ relative to the control group $(M=140 \mu \mathrm{g} / \mathrm{L}, S D=46)$, $F(1,27)=6.17, p<.05$.

Infants who developed significant postnatal conditions affecting growth and development $(n=3)$ or whose data was not available due to equipment failure $(n=1)$ were excluded from the analyses. Thus, the final sample consisted of 29 infants: 16 control infants ( 7 female) and 13 IDMs ( 7 female). The mean corrected age at the elicited imitation session was approximately 12 months (367 \pm 10 days; range 350-385) and there were no age differences between the groups $(p=.25)$. See Table 2 for a summary of group characteristics.

\footnotetext{
${ }^{1}$ Consistent with previous reports (Georgieff et al., 2002), postnatal follow-up assessments of iron status obtained between 6 and 12 months of age indicated that no infant born with low iron stores had iron deficiency at 1 year of age, as all measured ferritin concentrations were within the normal range $(21-88 \mu \mathrm{g} / \mathrm{L})$ and did not significantly differ between the two groups $(p=.89)$.
} 


\section{Materials}

Each test event was drawn from an existing pool of 11 different events (listed in Table 3) and counterbalanced across task and participants. The events consisted of two target actions that produced an interesting and desirable end state (example shown in Figure 1).

\section{Procedure}

All infants participated in two individual testing sessions that lasted approximately 1-hour each. Caregivers were present during both the testing sessions, but were not permitted to assist their children with the task. At the end of each session, the children received a small gift and caregivers were reimbursed for transportation costs. Ethical permission for the study was obtained from the recruitment hospitals and from the University of Minnesota; informed consent was obtained from the caregivers of the participating infants.

Elicited imitation-One of two trained researchers (authors TD and SW) conducted each elicited imitation session. After a period of free play, six different events were presented to the infant: two events that the infants were allowed to imitate immediately (the immediate imitation task), two events they were allowed to imitate after a 10-minute delay (the delayed imitation task), and two events in which the individual target actions of the two different events were presented interleaved with one another (the interleaved imitation task). See Table 4 for a schematic of the experimental design.

As in previous research, the immediate imitation task consisted of an infant-controlled baseline phase during which the infant was given general prompts (i.e., "What can you do with this stuff?"), followed by narrated modeling of the sequence two times in succession, and an opportunity for immediate imitation prompted by a verbal reminder (i.e., the name of the event sequence; see Bauer, in press). The delayed imitation task also consisted of a baseline phase, followed by modeling. However, a delay of approximately 10-minutes was imposed before the infant was allowed to imitate. The 10-minute delay imitation was "filled": during the delay the infants experienced the baseline and imitation phases of the immediate imitation task with different event sequences (Bauer et al., 1999). After the delay, the infants were given each set of props in turn and encouraged to imitate. The baseline phase served as a control for general problem solving skills or fortuitous production of the sequences and imitation served as the dependent measure of recall. Finally, the interleaved imitation task consisted of the alternating modeling of the two events, followed by imitation. No baseline measure was used in this task since it is an analogue to a working memory task and the cognitive processes of interest were the binding and integration of the information over time; if the elements of the sequence had been presented in advance of modeling, one could not have been certain that the processes were carried out during modeling.

Scoring-Infants' performance on the imitation tasks was videotaped and coded off-line by trained observers who were unaware of the group identity and the hypotheses of interest. Each task yielded two dependent measures: 1) the number of individual target actions produced, and 2) the ordered recall of those actions. In calculating the latter measure only the first occurrence of each target action was considered which reduced the likelihood that children will receive credit for production of a sequence by chance or by trial and error. For purposes of reliability $20 \%$ of the tapes were coded by a second trained observer. Mean percent agreement between the coders was $90.47 \%$ (range 70.59-100\%); when disagreements occurred, observations of the primary observer were used.

Bayley Scales of Infant Development-To assess overall developmental functioning the Bayley Scales of Infant Development, $2^{\text {nd }}$ Edition (BSID-II; Bayley, 1993) were administered to infants in both groups within approximately three weeks $(M=13, \mathrm{SD}=11$ days) of the 
elicited imitation session by one of two trained individuals (an occupational therapist and clinical psychology graduate student) who were unaware of the group status. Two scores were derived to index the infant's level of cognitive, language, personal-social, and fine and gross motor development: the mental development index (MDI) and the psychomotor developmental index (PDI).

Treatment of missing data-In a small number of cases $(n=4)$, data were missing at random (e.g., equipment failure, missed appointment). Due to the large individual differences in performance on these tasks and the interest regarding group differences, these data points were coded as missing and excluded from the analyses.

\section{Results}

\section{Preliminary Analyses}

Gestational age-On average, the infants in the IDM group were born earlier ( $M=38$, $S D=2$ weeks) than the infants in the control group $(M=40, S D=1$ week), $F(1,27)=7.21$, $p<.05$. Although this difference is not surprising (given that optimal management of the diabetic pregnancy is to deliver between 37 and 38 weeks; see Nold \& Georgieff, 2004), previous research with slightly older infants has suggested that memory performance may vary as a function of gestational age (see de Haan et al., 2000); therefore, this variable was entered as a covariate in all elicited imitation analyses.

Effects of overall cognitive development (Bayley Scales)—Results also indicated that there was a significant difference between the IDM and control group's performance on the MDI subscale, with IDMs $(M=95, S D=8)$ performing significantly below controls $(M$ $=103, S D=10): F(1,27)=5.50, p<.05$. Differences between the groups' performance on the PDI subscale was marginal, with the IDM group $(M=89, S D=21)$ performing below the control group $(M=102, S D=13: F(1,26)=3.93, p=.06)$. Since these results suggested differences in global cognitive functioning between the two groups, individual's MDI scores were also entered as a covariate in the analyses of elicited imitation performance in an attempt to isolate explicit memory performance from general cognitive ability.

Baseline elicited imitation measures-There was not a significant group difference in the baseline levels of performance for either dependent measure (all $p$ values $>.27$; Table 5, Panel A). Consequently, variation in long-term recall capabilities between the groups cannot be solely attributed to group differences in problem solving abilities.

\section{Recall Performance}

Across groups, paired samples t-tests revealed that infants learned and retained the information regarding both target actions and the order of the actions: For all three imitation tasks, performance was significantly greater during imitation than at baseline ${ }^{2}(p s<.05$; see Table 5 for descriptive statistics).

When effects of group on recall performance were investigated controlling for both gestational age and cognitive abilities, no significant group effects were found for production of target actions in the immediate, delayed, or interleaved imitation tasks or for production of pairs of target actions in the correct temporal order in either the immediate or interleaved imitation tasks (all $p \mathrm{~s}>.15$ ). However, the two groups performed differently on recall of target actions in the correct temporal order after a 10-minute delay: $F(1,22)=4.44, p<.05$. Thus, the IDM group did not produce fewer total actions than the control group, but the actions they did

\footnotetext{
${ }^{2}$ The average of the baseline for the immediate and delayed recall task was used for the interleaved imitation task.
} 
produce were not in the correct temporal order (see Figure 2). Specifically, 6 out of 16 controls produced more pairs of actions at recall than at baseline ( $\mathrm{p}<.05 ; 10$ ties), whereas only 1 out of 11 IDM infants produced more pairs of actions at recall than at baseline ( $\mathrm{p}=1.0 ; 9$ ties, 1 negative difference).

Finally, in order to determine if the differential performance on delayed imitation of pairs of actions between the groups was related to a specific prenatal risk, correlation analyses were conducted using newborn ferritin as a measure of prenatal iron deficiency and birth weight $\mathrm{z}$ scores as a measure of hypoxia and/or hyperinsulemia. Although birth weight $\mathrm{z}$ scores were not related to delayed recall $(p>.90)$, newborn ferritin concentration was marginally related to performance on delayed recall of actions in the correct temporal order: $r(27)=.36, p=.07$. Specifically, lower ferritin levels were associated with lower performance on temporally ordered recall in the delayed imitation task. Thus, an infant's iron status at birth, as indexed by ferritin, may address the extent of metabolic irregularity experienced prenatally and possible influences on behavioral outcomes 1 year later.

\section{Discussion}

The results of the present investigation indicate that even after differences in gestational age and global cognitive functioning are controlled, IDMs' delayed recall performance is below that of a control group at 1 year of age. This finding is consistent with the hypothesis that the risk factors accompanying the diabetic pregnancy alter explicit memory performance. Interestingly, this deficit appeared to be specific in that it altered temporally ordered recall only after a delay was imposed. Although IDMs' performance did not differ relative to controls on the immediate imitation and interleaved imitation tasks, and thus initially appeared asymptomatic, when memory demands were imposed via a delay, their performance began to decline relative to that of controls.

One experimental factor that may partially account for the differences in recall performance between the two groups was the filled delay: the infants were participating in another task while holding the to-be-recalled information in memory. Perhaps it is this additional demand of transferring and holding information in memory while completing another task with different materials that lead to the disparity between the groups: with the IDM group exhibiting more interference and/or forgetting than the control group. Although previous research has indicated that slightly older infants' (20-month-olds) performance on filled and unfilled delays did not differ (Bauer et al., 1999), the relative contributions of the delay itself and the potential interference caused by intervening events could not be disentangled in the present investigation. However, given the absence of effects for the interleaved task, there is at present no evidence to suggest this factor solely accounts for the differences in performance.

It is important to note that these behavioral differences were not the result of a preexisting discrepancy in the nature of the infants' interactions with test materials, due to the fact that the baseline measures did not differ between the groups. Nor can the results be attributed to differences in attention, motivation, or understanding of the task, as the two groups produced an equal number of target actions and the correct order of those actions when they were allowed to do so immediately after modeling (regardless of whether the presentation was blocked, as in the immediate imitation task, or interleaved). In short, such performance indicates that infants in the two groups were equally engaged in the tasks as well as willing and able to participate.

Results from this investigation are consistent with previous reports on older IDMs who present with poor cognitive outcomes on measures of general cognitive ability when they reach school age (e.g., Rizzo et al., 1997; Tamura et al., 2002). The present study utilized an assessment 
that possessed greater neural specificity than conventional developmental outcome measures, and controlled for variability in both general cognitive abilities and gestational age in order to investigate specific deficits in explicit mnemonic performance. Results suggest that deficits in explicit memory performance could not be solely attributable to differences in gestational age or general cognitive abilities. Furthermore, given that these measures were obtained at 1 year of age (as opposed to in later years) and that the infants were iron deficient in the pre- but not postnatal period (Georgieff et al., 2002), hypotheses regarding connections between prenatal experiences and behavioral outcome are more robust.

The marginal association between newborn ferritin concentration and behavioral recall on the delayed memory task suggested that prenatal iron status may be an important risk factor associated with cognitive outcome in the myriad factors related to the diabetic pregnancy. Animal models of prenatal iron deficiency suggest that this difference in performance may have arisen as a result of alterations in brain development during the prenatal period. Successful completion of the delayed imitation task is presumed to require the hippocampus to transfer encoded information from short-term storage to a more permanent status. Results of both animal and human research suggest that infants of diabetic mothers incur damage to this region (e.g., Jorgenson et al, 2003; Petry et al., 1992; Rao et al., 1999; 2003). Specifically, animal models have indicated that low levels of brain iron influence enzyme systems regulating brain growth, myelination, dopamine receptor synthesis, and energy metabolism, which may lead to adverse neurocognitive behavioral sequelae (e.g., for discussion and elaboration see Beard, 2003; de Ungria et al., 2000; Lozoff, 2000), and several investigations have suggested a selective influence of iron deficiency on neuronal energy metabolism in regions of the hippocampus and in the prefrontal cortex (deUngria et al., 2000; Rao et al., 2003), both of which are regions associated with explicit memory performance. Taken together, these studies suggest a relation between prenatal iron deficiency due to maternal diabetes and deficits in explicit memory performance.

In conclusion, the results of the present investigation are consistent with the hypotheses that risk factors associated with the diabetic pregnancy alter prenatal development, which can influence memory performance on a delay recall task. Future investigations should attempt to further isolate contributions of the independent risk factors (e.g., prenatal iron deficiency) and their combined influence to illuminate their impact on neurobehavioral outcomes and explore their influence across the life span.

\section{Acknowledgments}

This research was supported by grants from the National Institute of Health to Charles A. Nelson (NS34458), Michael K. Georgieff (HD29421), and Patricia J. Bauer (HD-28425); and by a grant from the NIH National Center for Research Resources (RR00400). We are grateful to the members of the Center for Neurobehavioral Development, Developmental Cognitive Neuroscience Laboratory, and Cognition in the Transition Laboratory (in particular Jennifer Haight), who provided instrumental support with this research project. We are also thankful to the families who participated in this research. Portions of these data were displayed at the meeting for the International Society for Infant Studies in Toronto, Canada, April 2002 and at the meeting for Society for Research in Child Development in Tampa Bay, FL, April 2003.

\section{References}

Adlam A-LR, Vargha-Khadem F, Mishkin M, de Haan M. Deferred imitation of action sequences in developmental amnesia. J Cogn Neurosci. in press.

Akin M, Ceran O, Atay E, Atay Z, Akin F, Akturk Z. Postpartum maternal levels of hemoglobin A1c and cord C-peptide in macrosomic infants of non-diabetic mothers. J Matern Fetal Neonatal Med 2002;12:274-276. [PubMed: 12572597] 
Barks JD, Sun R, Malinak C, Silverstein FS. gp120, an HIV-1 protein, increases susceptibility to hypoglycemic and ischemic brain injury in perinatal rats. Exp Neurol 1995;132:123-133. [PubMed: 7720821]

Bauer, PJ. New developments in the study of infant memory. In: Teti, DM., editor. Handbook of Research Methods in Developmental Psychology. Blackwell Publishers; Oxford, United Kingdom: in pressTo appear in

Bauer PJ, Van Abbema DL, de Haan M. In for the short haul: Immediate and short-term remembering and forgetting by 20-month-old children. Infant Behav Dev 1999;22:321-343.

Bayley, N. Bayley Scales of Infant Development. 2nd. Ed.. The Psychological Corporation; San Antonio: 1993.

Beard J. Iron deficiency alters brain development and functioning. J Nutr 2003:1468S-1472S. [PubMed: 12730445]

de Haan M, Bauer PJ, Georgieff MK, Nelson CA. Explicit memory in low-risk infants aged 19 months born between 27-42 weeks of Gestation. Dev Med Child Neurol 2000;42:304-312. [PubMed: 10855650]

de Ungria M, Rao R, Wobken JD, Luciana M, Nelson CA, Georgieff MK. Perinatal iron deficiency decreases cytochrome c oxidase (CytOx) activity in selective regions of neonatal rat brain. Pediatr Res 2000;48:169-176. [PubMed: 10926291]

DeBoer, T.; Georgieff, MK.; Nelson, CA. Declarative memory performance in infants of diabetic mothers. In: Bauer, Patricia J., editor. Varieties of early experience: Influences on declarative memory development. Lawrence Erlbaum Associates; 2004. Submitted for publication in

deRegnier R-A, Nelson CA, Thomas K, Wewerka S, Georgieff MK. Neurophysiologic evaluation of auditory recognition memory in healthy newborn infants and infants of diabetic mothers. J Pediatr 2000;137:777-784. [PubMed: 11113833]

Georgieff, MK.; Rao, R. The role of nutrition in cognitive development. In: Nelson, CA.; Luciana, M., editors. Handbook of developmental cognitive neuroscience. The MIT Press; Cambridge, MA: 2001. p. $45-58$.

Georgieff MK, Wewerka SW, Nelson CA, deRegnier R-A. Iron status of infants with low iron stores at birth. J Pediatr 2002;141:405-409. [PubMed: 12219063]

Hannonen R, Tupola S, Ahonen T, Riikonen R. Neurocognitive functioning in children with type-1 diabetes with and without episodes of severe hypoglycaemia. Dev Med Child Neurol 2003;45:262268. [PubMed: 12647928]

Jorgenson LA, Wobken JD, Georgieff MK. Perinatal iron deficiency alters apical dendritic growth in hippocampal CA1 pyramidal neurons. Dev Neurosci 2003;25:412-420. [PubMed: 14966382]

Low JA, Galbraith RS, Muir DW, Killen HL, Pater EA, Karchmar EJ. Factors associated with motor and cognitive deficits in children after intrapartum fetal hypoxia. Am J Obstet Gynecol 1984;148:533539. [PubMed: 6199975]

Lozoff B. Perinatal iron deficiency and the developing brain. Pediatr Res 2000;48:137-139. [PubMed: 10926285]

McDonough L, Mandler JM, McKee RD, Squire LR. The deferred imitation task as a nonverbal measure of declarative memory. Proc Natl Acad Sci USA 1995;92:7580-7584. [PubMed: 7638234]

Morris MA, Grandis AS, Litton JC. Glycosylated hemoglobin concentration in early gestation associated with neonatal outcome. Am J Obstet Gynecol 1985;153:651-654. [PubMed: 4061535]

Nelson C, Silverstein FS. Acute disruption of cytochrome oxidase activity in brain in a perinatal rat stroke model. Pediatr Res 1994;36:12-19. [PubMed: 7936831]

Nelson CA, Wewerka SS, Borscheid AJ, deRegnier R-A, Georgieff MK. Electrophysiologic evidence of impaired cross-modal recognition memory in 8-month-old infants of diabetic mothers. J Pediatr 2003;142:575-582. [PubMed: 12756394]

Nelson CA, Wewerka S, Thomas KM, Tribby-Walbridge S, deRegnier R-A, Georgieff M. Neurocognitive sequelae of infants of diabetic mothers. Behav Neurosci 2000;114:950-956. [PubMed: 11085609]

Nold J, Georgieff M. Infants of diabetic mothers. Pediatr Clin North Am 2004;51:619-637. [PubMed: 15157588] 
Petry CD, Eaton MA, Wobken JD, Mills MM, Johnson DE, Georgieff MK. Iron deficiency of liver, heart, and brain in newborn infants of diabetic mothers. J Pediatr 1992;121:109-114. [PubMed: 1625067]

Rao R, de Ungria M, Sullivan D, Wu P, Wobken JD, Nelson CA, Georgieff MK. Perinatal iron deficiency increases the vulnerability of rat hippocampus to hypoxic ischemic insult. J Nutr 1999;129:199-206. [PubMed: 9915900]

Rao R, Tkac I, Townsend EL, Gruetter R, Georgieff MK. Perinatal iron deficiency alters the neurochemical profile of the developing rat hippocampus. J Nutr 2003;133:3215-3221. [PubMed: 14519813]

Rizzo TA, Metzger BE, Dooley SL, Cho NH. Early malnutrition and child neurobehavioral development: Insights from the study of children of diabetic mothers. Child Dev 1997;68:26-38. [PubMed: 9084122]

Siddappa AM, Georgieff MK, Wewerka S, Worwa C, Nelson CA, deRegnier R-A. Iron deficiency alters auditory recognition memory in newborn infants of diabetic mothers. Pediatr Res 2004;55:10341041. [PubMed: 15155871]

Tamura T, Goldenberg RL, Hou J, Johnston KE, Cliver SP, Ramey SL, Nelson KG. Cord serum ferritin concentrations and mental and psychomotor development of children at five years of age. J Pediatr 2002;140:165-170. [PubMed: 11865266]

Widness JA, Susa JB, Garcia JF, Singer OB, Sehgal P, Oh W, Schwartz R, Schwartz HC. Increased erythropoiesis and elevated erythropoietin in infants born to diabetic mothers and in hyperinsulinemic rhesus fetuses. J Clin Invest 1981;67:637-642. [PubMed: 7009647] 

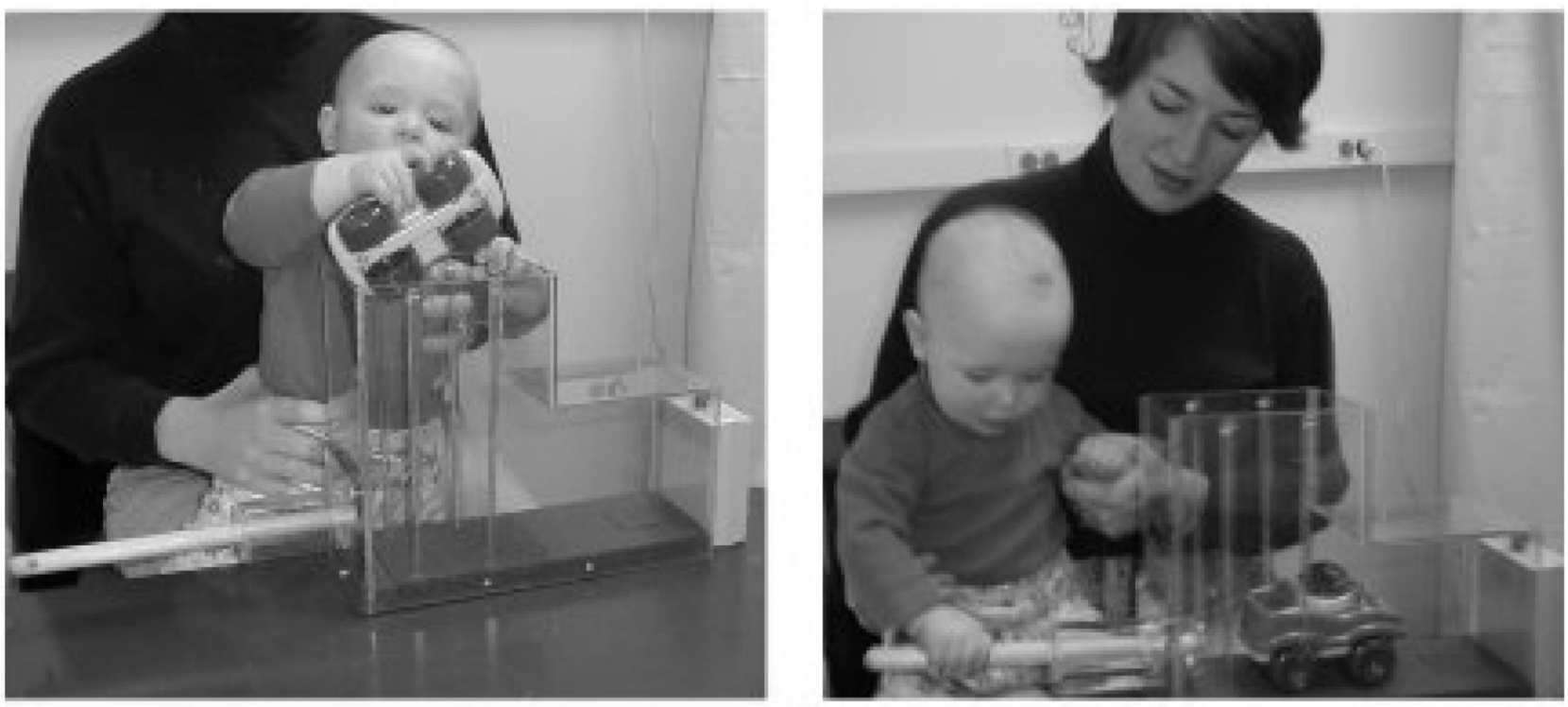

Figure 1.

Photographs of infant completing the event sequence "Turn on the light." To complete the first step, the infant places a small car into the track of an L-shaped apparatus. To ultimately realize the end state of the event, the infant pushes a plunger, thereby causing the car to travel down the track, tripping a small switch that causes a light to illuminate. (Obtaining permission to reprint from Psychological Science.) 
A

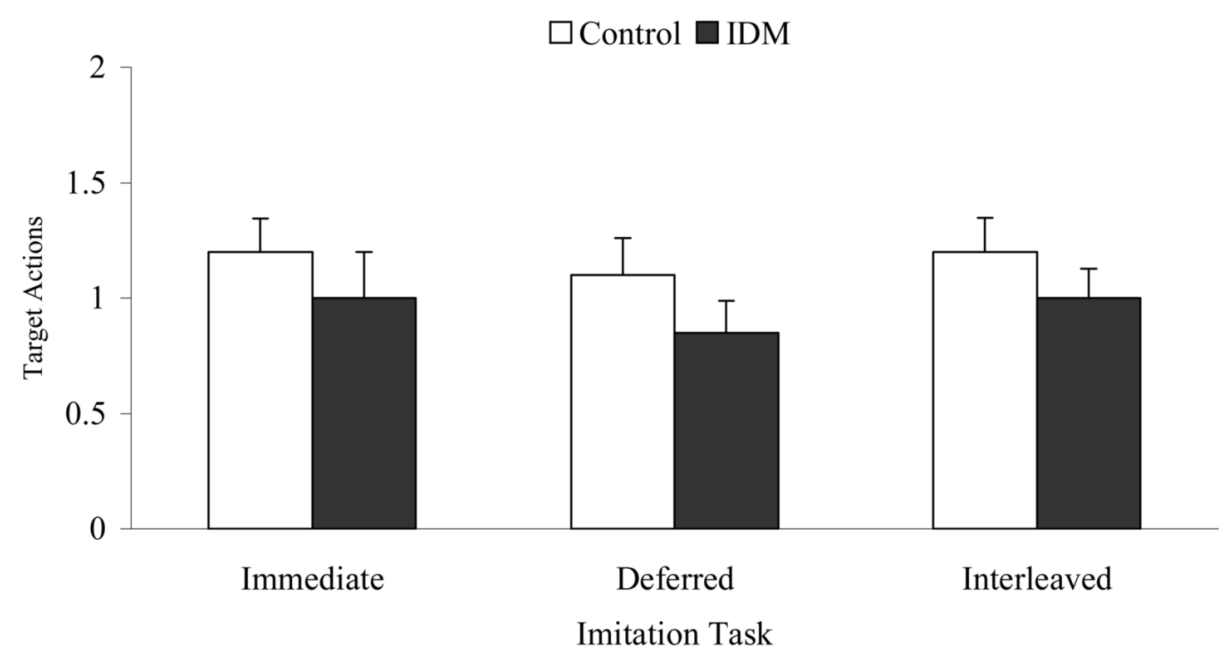

B

$\square$ Control $\square$ IDM

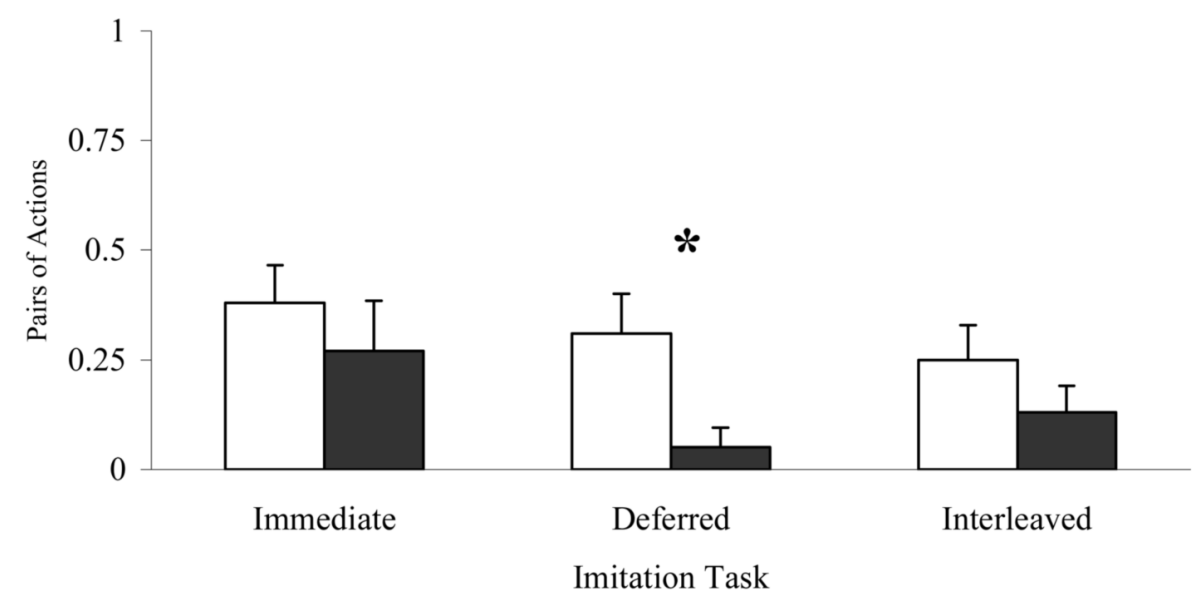

* denotes $p \leq .05$

Figure 2.

Mean (+1 SEM) number of target actions (A) and correctly ordered pairs of actions (B) produced during immediate, 10-minute delayed, and interleaved recall by control and IDM children. 
Table 1

\title{
Medical Criteria for Inclusion
}

\author{
1. No pregnancy complications \\ - Lack of intrauterine growth restriction, significant maternal hypertension, chromosomal syndromes/non- \\ chromosomal congenital anomalad sequences, and congenital infectious agents (TORCH) \\ 2. Normal labor and delivery \\ - $\quad$ No significant heart rate decelerations and 5 minute Apgar scores $>6$ \\ 3. Uncomplicated neonatal course \\ - No mechanical ventilation and no indication of acute perinatal or neonatal insult such as asphyxia, sepsis, \\ seizures, meningitis, or intracranial hemorrhage
}


Table 2

Characteristics of the Infants of Diabetic Mothers (IDM) and Control Groups

\begin{tabular}{lccc}
\hline Characteristic & $\begin{array}{c}\text { Control } \\
\text { Mean }(\text { SD) }\end{array}$ & $\begin{array}{c}\text { IDM } \\
\text { Mean }(\text { SD) })\end{array}$ & $\begin{array}{c}\text { F statistic of one-way } \\
\text { ANOVA with group as } \\
\text { a factor or Chi Square statistic }\end{array}$ \\
\hline Gestational age at birth (wk) & $40(1)$ & $38(2)$ & $F(1,27)=7.21, p<.05$ \\
Birth weight $(\mathrm{g})$ & $3639(348)$ & $3797(947)$ & $n s$ \\
$\quad$ Birth weight $z$ scores & $.43(.75)$ & $1.8(2.1)$ & $F(1,27)=6.05, p<.05$ \\
$\quad$ Birth weight $z$ scores $>2$ & $0 / 16$ & $7 / 13$ & $\chi^{2}(1, N=29)=11.36, p<.01$ \\
Prenatal (newborn) Ferritin $(\mu \mathrm{g} / \mathrm{L})$ & $140(46)$ & $87(68)$ & $F(1,27)=6.17, p<.05$ \\
$\quad$ Ferritin $\leq 76 \mu \mathrm{g} / \mathrm{L}$ & $0 / 16$ & $6 / 13$ & $\chi^{2}(1, N=29)=9.31, p<.01$ \\
Postnatal (6-12 month) Ferritin $(\mu \mathrm{g} / \mathrm{L})$ & $46(21)$ & $48(26)$ & $n s$ \\
Age at test (days) & $365(11)$ & $379(9)$ & $n s$ \\
Age at Bayley (days) & $380(14)$ & $379(11)$ & $n s$ \\
Bayley Scales: MDI & $103(10)$ & $95(8)$ & $F(1,27)=5.50, p<.05$ \\
Bayley Scales: PDI & $102(13)$ & $89(21)$ & $F(1,26)=3.93, p=.06$ \\
\hline
\end{tabular}


Table 3

Sequences Used for the Elicited Imitation Task

\begin{tabular}{cccc}
\hline Event Name & $\mathbf{1}^{\text {st }}$ Target Action & $\mathbf{2}^{\text {nd }}$ Target Action & End State \\
\hline Make a Glowball & "Open the lid" & "Pull out the drawer" & Glowball lights up \\
Make a Gong & "Hang up the bell" & "Ring it" & Gong rings \\
Turn on the light & "Put in the car" & "Push the stick" & Light turns on \\
Find Bubbles & "Put in the block" & "Push it in" & Bubbles pops out \\
Make a Happy Face & "Open the door" & "Push in the block" & Happy face appears \\
Find the Bear & "Slide the bar" & "Open the door" & Bear appears \\
Make a Balloon & "Put in the balloon" & "Press it" & Balloon blows up \\
Make an Airplane & "Unfold it" & "Fly it" & Airplane flies \\
Make a Rattle & "Cover it" & "Shake it" & Rattle shakes \\
Make a Jumper & "Push in the ball" & "Pop it" & Ball jumps \\
Go for a Duck Walk & "Put down the ramp" & "Go for a walk" & Duck slides down ramp \\
\hline
\end{tabular}


Table 4

Presentation of Event Sequences

\begin{tabular}{lll}
\hline Baseline & Modeling & Recall \\
\hline Delay 1 & Delay 1 & \\
Delay 2 & Delay 2 & Immediate 1 \\
Immediate 1 & Immediate 1 & Immediate 2 \\
Immediate 2 & Immediate 2 & Delay 1 \\
& & Delay 2 \\
& Interleaved 1-1 & \\
& Interleaved 2-1 & \\
& Interleaved 1-2 & Interleaved 1 \\
& Interleaved 2-2 & Interleaved 2 \\
\hline
\end{tabular}

Note: Infants were presented with 6 different event sequences. For two of the sequences infants were allowed to imitate immediately after baseline and modeling (Immediate 1 and 2), for two of the sequences a 10-minute delay was imposed before imitation was allowed (Delay 1 and 2), and presentation of the remaining two sequences was interleaved such that infants saw the first step of Sequence 1, the first step of Sequence 2 (Interleaved 1-1, Interleaved 1-1), then the second step of Sequence 1 and the second step of Sequence 2 (Interleaved1-2, Interleaved 2-2) before imitation was permitted. 


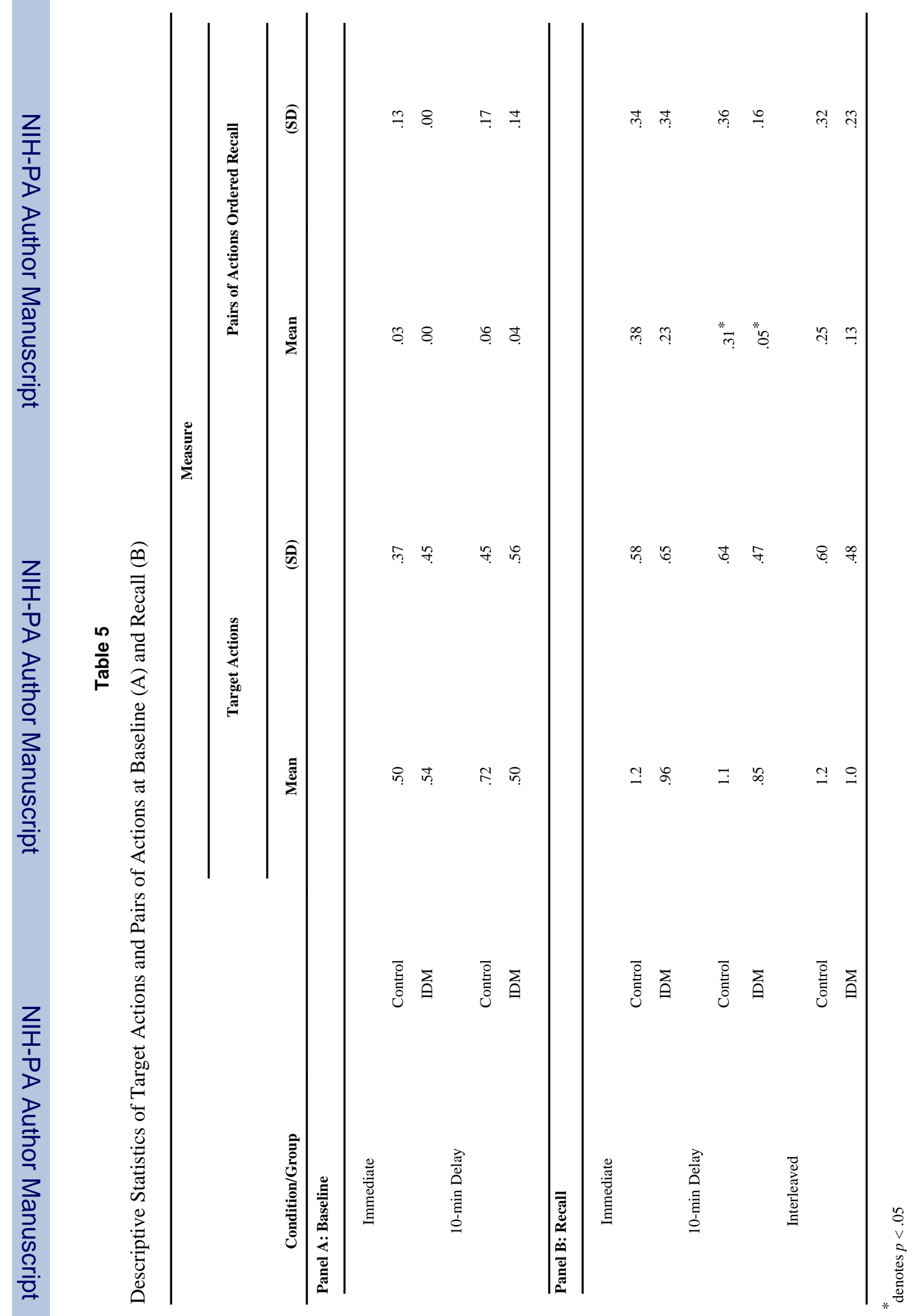

